\title{
Aesthetic defect characterization of a polymeric polarizer via structured light illumination
}

\author{
Wen-wei LAI, Xiao-xing ZENG, Jian HE, Yuan-long DENG* \\ (College of Mechatronics and control Engineering, Shenzhen university, Shenzhen \\ 518060, China) \\ * Corresponding author: dengyl@ szu.edu.cn
}

\begin{abstract}
The aesthetic defects of polymeric polarizers have a serious impact on the quality of thin film transistor liquid crystal display (TFT-LCD) panels. However, some of these slight and transparent defects can barely be imaged and characterized using conventional illumination. To inspect these special defects, a new and automated inspection method is proposed that employs structured-light illumination. A machine vision system that uses a LCD monitor to produce a binary stripe pattern was designed to enhance the imaging defects. Thus, subsequent image processing is straightforward and simple because of this enhancement. One hundred and fifty defect samples were successfully imaged and then processed and characterized by the Robust Principal Component Analysis (RPCA) algorithm. The total inspection accuracy was up to $99 \%$. The experiments demonstrate that the width of the luminous stripe should be optimized at 2 to 3 times the size of the defect. The mechanism of the imaging enhancement is preliminarily discussed.
\end{abstract}

Keywords: Machine vision; Defect inspection; Structured-light illumination; Polymeric polarizer; RPCA algorithm; Transparent defects.

\section{Introduction}

As a standard polarizing optical component, the polymeric polarizer has a wide range of applications. For instance, two pieces of polymeric polarizer with orthogonal polarization directions are indispensable in TFT-LCD panels. The polarizer used in the TFT-LCD panels typically consists of six layers of polymeric films including a layer of pressure-sensitive adhesive (PSA), as illustrated in Fig. 1. Each layer is tens of micrometers. The polyvinyl alcohol (PVA) is surrounded by triacetyl cellulose (TAC) and must be polarized. The TAC can isolate PVA from air and humidity. A separate film (SF) protects the PSA.

Fig. 1. Schematic of polymeric polarizer. PF: Protective Film, TAC: Triacetyl 
Cellulose, PVA: Polyvinyl Alcohol, PSA: Pressure Sensitive Adhesive, and SF: Separate Film.

For a polymeric polarizer, imperfections in the materials and failures during manufacturing will produce aesthetic defects that can be visually perceived by end users. These tiny defects are aesthetically displeasing but do not have any functional disadvantages.

This paper characterizes 80 to several hundreds of micrometer aesthetic defects via human sight and human sight resolution limitations. There are many kinds of aesthetic defects including impurities, stains, scratches, dents and bubbles. These could occur at any layer of the polymeric polarizer. In addition, the classification would be more complicated if the location, size and shape of a defect are considered. Most aesthetic defects are located inside the polymeric polarizer and not on the surface.

Currently, most research on polymeric polarizers is focused on the polarizer's optical features including reflectivity, transmissivity and extinction ratio. The aesthetic defects are almost neglected, even although they actually play an even more important role in quality assurance [1]. Visual inspection by humans is widely adopted in the manufacture of both polymeric polarizers and TFT-LCD panels. This manual work is quite labor intensive, and the inspection accuracy is influenced by subjective nature of the human inspectors - they can be tired or have poor eyesight. Furthermore, the speed of manual inspection is difficult to improve. For instance, it will take at least one minute for an experienced inspector to examine a 19-inch piece of polymeric polarizer. Hence, an automated and non-destructive detection technology with high accuracy and speed for such defects is quite important for quality assurance on production lines.

Machine vision has been widely used to inspect product defects. Jerjen et al. used X-ray Dark Field Imaging to visualize and detect the stress whitening of an injection-molded polyvinylidene fluoride [2]. Chou et al. designed a machine vision system based on a linear array CCD camera to detect the aesthetic defects of polymeric polarizer, and also studied applications of Down-sampling Compression, Wavelet Transform and Hough Transform [3]. Yeh and $\mathrm{Wu}$ developed an image-processing filter to enhance the defects for a higher inspection speed based on 
a Standard Deviation Algorithm [4]. Tsai proposed an improved rapid density-based clustering algorithm and used adaptive threshold values to segment the polarizer images [5].

There are some special aesthetic defects of the polymeric polarizer we report here for the first time which have nearly identical reflectivity and transmissivity with the normal region. These defects are difficult to image under conventional illumination. Thus, we propose to define them as "transparent defects" such as dents, transparent impurities and bubbles. These transparent defects are the main obstacle to the existing detection technology of the polymeric polarizer because of their extremely low contrast.

Structured-light imaging is mainly used for 3D rebuilding and 3D surface measurements [6-7]. Geng delivered an overall description and detailed discussion of structured-light imaging [8]. For higher accuracy and speed, current research of structured-light imaging is mainly focused on the algorithms of calibrating projects and cameras, mapping spatial coordinate systems, coding of surface spots, and phase demodulation [9-11]. Seulin et al. proposed a new method to detect the surface defects on a specular object by employing structured-light illumination in 2002. This report offers theoretical model and experimental results [12]. Aluze et al. employed structured lighting to detect the surface defects of a glass bottle and modeled the defects as surface bulges and dents to explain the imaging enhancement [13]. Martínze et al. employed structured lighting to detect the surface defects of a non-plane transparent headlamp lens. Defects appeared in the captured image as a set of luminous pixels in a dark zone - thus, the contrast was very good. The adaptive threshold segmentation algorithm was studied based on the aspect images to improve the detection accuracy and speed [14-15]. Macher and Gruber used phase measuring deflectometry with structured lighting to detect visually perceptible sink marks - a type of surface defects - on high gloss injection molded parts. They then modeled the surface defects for fast measurements of their visual perceptibility [16-17].

Here, we propose a new method to detect these inner transparent defects. The contrast of the defects was markedly enhanced under structured-light illumination in favor of better detection accuracy and speed. This scheme used a machine vision inspection system, and the validity of this new method was verified via experimental 
results.

\section{Experimental setup}

The machine vision inspection system is illustrated in Fig. 2. A LCD monitor served as a structured-light source to display the binary stripe pattern (Fig. 3). The pattern was produced and controlled by a window application. Thus, the widths of the stripes could be easily adjusted. The resolution of the camera and the structured-light source were $2592 * 1944$ and $2048 * 1536$, respectively. The pattern moved $1 / 4$ of the width of the stripe each time, and the camera then captured a picture of the sample that was illuminated by the structured-light source. Thus, it would take four steps to cover the entire sample. This is called active light scanning without any mechanical movements. The computer processed the four static pictures captured in each step and identified the defects of the sample. More scanning steps per sample offer better inspection accuracy but longer scan times.

Fig. 2. Defect inspection system.

Fig. 3. Linear binary structured-light pattern.

\section{Imaging results and analysis}

The contrast of defect imaging is defined as:

$$
V=\frac{\left|G_{B}-G_{D}\right|_{\max }}{G_{B}+G_{D}} \times 100 \%
$$

Here, $G_{D}$ and $G_{B}$ are the gray levels of the defect and the adjacent area, respectively. There were three samples with defects located in the marking circles: No. 1 with a dent, No. 2 with a transparent impurity and No. 3 with an air bubble. Fig. 4 shows these images under uniform illumination and structured-light illumination; the inserts are the enlarged local images. Under uniform illumination, the contrast of the defects is approximately equal to zero, as illustrated in Fig. 4a, Fig. 4c and Fig. 4e, regardless of optimization parameters used in the inspection system. This issue is the biggest challenge in detecting aesthetic defects in a polymeric polarizer. In contrast, the defects appear considerably clearer under structured-light illumination (Fig. 4b, Fig. 4d, and Fig. 4f). The contrast of the defects markedly improves to $68.0 \%, 88.2 \%$, and $31.5 \%$, respectively. It is believable that the imaging enhancement resulting from the 
structured lighting would markedly simplify the subsequent image-processing algorithm, and be very beneficial to improve the inspection accuracy and speed.

Fig. 4. Transparent defects in the sample No. 1 3 as seen by (a), (c), and (e) uniform illumination imaging; (b), (d), and (f) structured lighting imaging.

According to Fig. 5, the effect of the imaging enhancement partially depends on the stripe width. The best contrast of the defect in Fig. 5c and Fig. 5d both reach up to $100 \%$. When the stripe width is $0.5 \mathrm{~mm}$ (Fig. 5c), all luminous zones can be detected, i.e., the defects located in the luminous regions can be distinctly imaged and detected. In contrast, only the edge regions in Fig. 5d can enhance the imaging of the transparent defects, i.e., a defect can not be imaged when it is located in the luminous zones or the dark zones because of the increasing stripe width. In addition, the luminous zones and the dark zones in Fig. 5d work similarly to uniform illumination. Therefore, the stripe width should be optimized at two to three times the size of the defect because image enhancement and inspection efficiency are simultaneously considered.

Fig. 5. Imaging enhancement with different stripe widths. (a) Uniform illumination; (b) Stripe width $0.2 \mathrm{~mm}$; (c) Stripe width $0.5 \mathrm{~mm}$; (d) Stripe width $3.5 \mathrm{~mm}$.

\section{Image processing and inspection results}

PCA is a popular tool for high-dimensional data analysis with applications in many scientific and engineering fields [18]. We propose two algorithms to solve the convex optimization problem of PCA: the Accelerated Proximal Gradient (APG) [19] and Inexact Augmented Lagrange Multiplier (IALM) [20]. The APG and IALM can be directly applied to the original images such as Fig. 4 without any preprocessing because of the image enhancement and robustness of RPCA. These two RPCA algorithms were implemented in LabVIEW and Vision Development Module (VDM). For the defects shown in Fig. 4b, Fig. 6a and Fig. $6 \mathrm{~b}$ show the results produced by APG and IALM, respectively. They do not need segmentation to the binary images because the target defects have satisfactory contrast. The defect features, including the locations, shapes and sizes, can be directly extracted using the CountObjects2 function of VDM (Fig. 6c and Fig. 6d). Thus, the effects of APG and IALM are basically the same. The same procedure worked on sample No. 2, as illustrated in Fig. 4d, which led to Fig. 7. The transparent impurity was also correctly identified. The 
validity of this inspection approach was further verified.

Fig. 6. Inspection results of sample No. 1. (a) Result of APG, (b) Result of IALM, (c) Defect extraction from (a), (d) Defects extraction from (b).

Fig. 7. Inspection results of sample No. 2. (a) Result of APG, (b) Result of IALM, (c) Defects extraction from (a), (d) Defects extraction from (b).

One hundred and fifty samples were imaged under structured-light illumination and processed by APG and IALM, respectively. The inspection accuracy was up to 99\% (Table 1). The accuracies of the two algorithms are basically the same if the defects are enhanced by structured lighting.

Tab. 1 Inspection results of 105 aesthetic defects.

\begin{tabular}{c|ccc}
\hline & Correct & False & Undetected \\
\hline APG & 104 & 1 & 0 \\
IALM & 104 & 0 & 1 \\
\hline
\end{tabular}

\section{Discuss of enhancement mechanism}

It is very important to understand why imaging of the transparent aesthetic defects is greatly enhanced under structured lighting. In general, structured-light imaging means a surface is illuminated by a light source with a 2D and spatially varying intensity pattern. If the scene is a planar surface without any $3 \mathrm{D}$ surface variation, then the pattern shown in the acquired image is similar to that of the projected structured-light pattern. However, when the surface in the scene is non-planar, the geometric shape of the surface distorts the projected structured-light pattern as seen from the camera. The principle of structured-light 3D surface imaging techniques is to extract the $3 \mathrm{D}$ surface shape based on the information from the distortion of the projected structured-light pattern. Accurate 3D surface profiles of objects in the scene can be computed with various structured-light principles and algorithms [8]. Clearly, this principle of structured-light imaging does not work with the image enhancement scheme studied here.

The defect model and imaging mechanism built by Seulin in 2002 were sequentially adopted by other researchers [13-17]. They modeled the surface defects of specular objects as bulges and dents. Thus, the slope variation of the defect region altered the normal direction of the reflection plane. Thus, a surface defect appeared in 
the captured image as a clear spot in a dark zone or a dark spot in a luminous zone - this had good contrast [12]. A similar phenomenon occurred in our experiments, i.e., a set of luminous pixels in a dark zone or vice versa. However, imaging of the inner transparent defect does not completely follow the model and the mechanism.

For instance, we found that the image distance plays a very important role in imaging the transparent aesthetic defects. Here the image distance is defined as the distance between the sample and the camera. Fig. 8 illustrates the images of a defect at different image distances. The defect region is brighter than background at $107 \mathrm{~mm}$ (Fig. 8a). At $113 \mathrm{~mm}$ and $120 \mathrm{~mm}$, the defect appears the same and darker, respectively. Fig. 9 further demonstrates that the gray level difference between the defects with background varies with image distance. More experiments verified the same rule in which the defect images gradually shift from brighter than background to the same. They then become darker with increased image distance. In conclusion, the image enhancement of the transparent defects is partially determined by the image distance. The defect model and imaging mechanism discussed in Refs. 12-17 cannot explain these experimental results (Fig. 8 and Fig. 9). To optimize the design of the inspection system, the imaging enhancement mechanism requires further study.

Fig. 8. Defect imaging at different image distances. (a) $107 \mathrm{~mm}$, (b) $113 \mathrm{~mm}$, (c) 120 $\mathrm{mm}$.

Fig. 9. Gray level difference versus image distance.

\section{Conclusions}

The transparent aesthetic defects inside a polymeric polarizer have low contrast that is even invisible under common illumination. To detect these defects, a new method is proposed based on active structured lighting that visualizes these invisible defects. A machine vision system without any mechanical moving devices was built that employs a LCD monitor to display the programmable structured light pattern. Image processing is straightforward and simple. The width of the luminous stripe should be about 2 to 3 times the size of the defect if the defect contrast and the detection efficiency are considered concurrently. The experimental results verify the validity of this new method. It should be specially noted there are other defects in the images given above, such as dust, stains, and scratches. These non-original defects are 
produced during our repeated experiments and can also be detected with this new method. To optimize the design of the inspection system, the image enhancement mechanism requires further study.

\section{Acknowledgements}

We gratefully acknowledge Shenzhen SAPO Photoelectric Co., Ltd for preparing the polarizer samples. This work is supported in part by the National Science Foundation of China under Grants 61571306, and by the Basic Research Program Funds of Shenzhen under Grant JCYJ20150324141711676.

\section{References}

[1] L. Zsiros, A. Suplicz, G. Romhany, T. Tabi, J.G. Kovacs, Development of a novel color inhomogeneity test method for injection molded parts, Polymer Testing 37 (2014) 112-116.

[2] I.Jerjen, V.Revol, A.J. Brunner, P.Schuetz, C. Kottler, R. Kaufmann, T. Luethi, G. Nicoletti, C. Urban, U. Sennhauser, Detection of stress whitening in plastics with the help of X-ray dark field imaging, Polymer Testing 32 (2013) 1094-1098.

[3] C. F. J. Kuo, C. H. Chiu, Y. C. Chou, Research and development of intelligent on-line real-time defect inspection system for polymer polarizer, Polymer-Plastics Technology and Engineering 48 (2009) 185-192.

[4] C. H. Yeh, F. C. Wu, An image enhancement technique in inspecting visual defects of polarizers in TFT-LCD industry, IEEE International Conference on Computer Modeling and Simulation, ICCMS (2009) 257-261.

[5] M. Tsai, C. Wu, F. Tian, Development of an improved rapid density-based clustering algorithm and its application on polarizer defect inspection, Journal of Technology, 24 (2009) 267-274.( in Chinese)

[6] F. Alhwarin, A. Ferrein, I. Scholl, IR Stereo Kinect: Improving depth images by combining structured light with IR stereo, Lecture Notes in Computer Science, 8862 (2014) 409-421.

[7] X. Chen, Y.H. Yang, Scene adaptive structured light using error detection and correction, Pattern Recognition, 48 (2015) 220-230.

[8] J. Geng, Structured-light 3D surface imaging: a tutorial, Advances in optics and photonics, 3 (2011) 128-160.

[9] Q. Sun, Y. Hou, Q. Tan, A Flexible Calibration Method Using the Planar Target with a Square Pattern for Line Structured Light Vision System, PLoS ONE, 9 (2014) e106911.

[10] B. Li, S. Zhang, Structured light system calibration method with optimal fringe angle, Applied Optics, 53 (2014) 7942-7950.

[11] K. Liu, X. Luo, W. Wu, Fast and accurate calibration algorithm for laser pointer tracking via structured light illumination, Electronics Letters 50 (2014) 1946-1948.

[12] R. Seulin, F. Merienne, P. Gorria, Simulation of specular surface imaging based 
on computer graphics: Application on a vision inspection system, EURASIP Journal on Applied Signal Processing 7 (2002) 649-658.

[13] D. Aluze, F. Merienne, C. Dumont, P. Gorria, Vision system for defect imaging, detection, and characterization on a specular surface of a 3D object; Image Vision Computing 20 (2002) 569-580.

[14] Satorres Martínez, S., Gómez Ortega, J., Gámez García, J.,Sánchez García, A., An industrial vision system for surface quality inspection of transparent parts, Int $\mathbf{J}$ Adv Manuf Technol 68 (2013) 1123-1136.

[15] Satorres Martínez, S., Gómez Ortega, J., Gámez García, J.,Sánchez García, A., A machine vision system for defect characterization on transparent parts with non-plane surfaces, Machine Vision and Applications 23 (2012) 1-13.

[16] J.Macher, D. P. Gruber, T. Altenbuchner,G.A. Pacher, G.R. Berger, W. Friesenbichler, Detection of visually perceptible sink marks on high gloss injection molded parts by phase measuring deflectometry, Polymer Testing 34 (2014) 42-48.

[17] D. P. Gruber, J. Macher, D. Haba, G. R. Berger,Ge. Pacher, W. Friesenbichler, Measurement of the visual perceptibility of sink marks on injection molding parts by a new fast processing model, Polymer Testing 33 (2014) 7-12.

[18] C.Y. Wang, S.Y. Qin, Adaptive detection method of infrared small target based on target-background separation via robust principal component analysis, Infrared physics \& technology 69(2015) 123-135.

[19] Kim-Chuan Toh, Sangwoon Yun, An accelerated proximal gradient algorithm for nuclear norm regularized least squares problems, Pacific Journal of Optimization 6 (2010) 615-640.

[20] Y. Xu, X.Z. Fang, J. Wu, X.1. Li, D. Zhang, Discriminative Transfer Subspace Learning via Low-Rank and Sparse Representation, IEEE Transactions on Image Processing 25(2016) 850-63. 


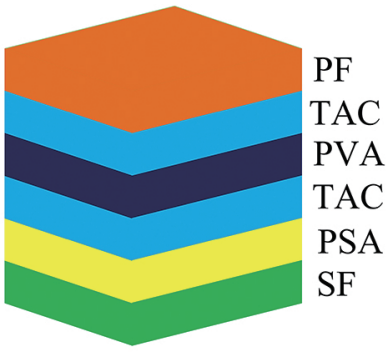




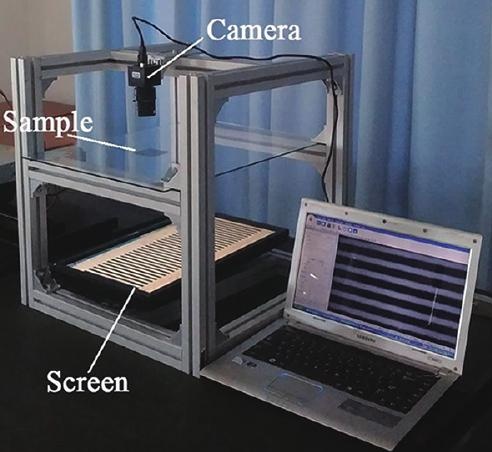





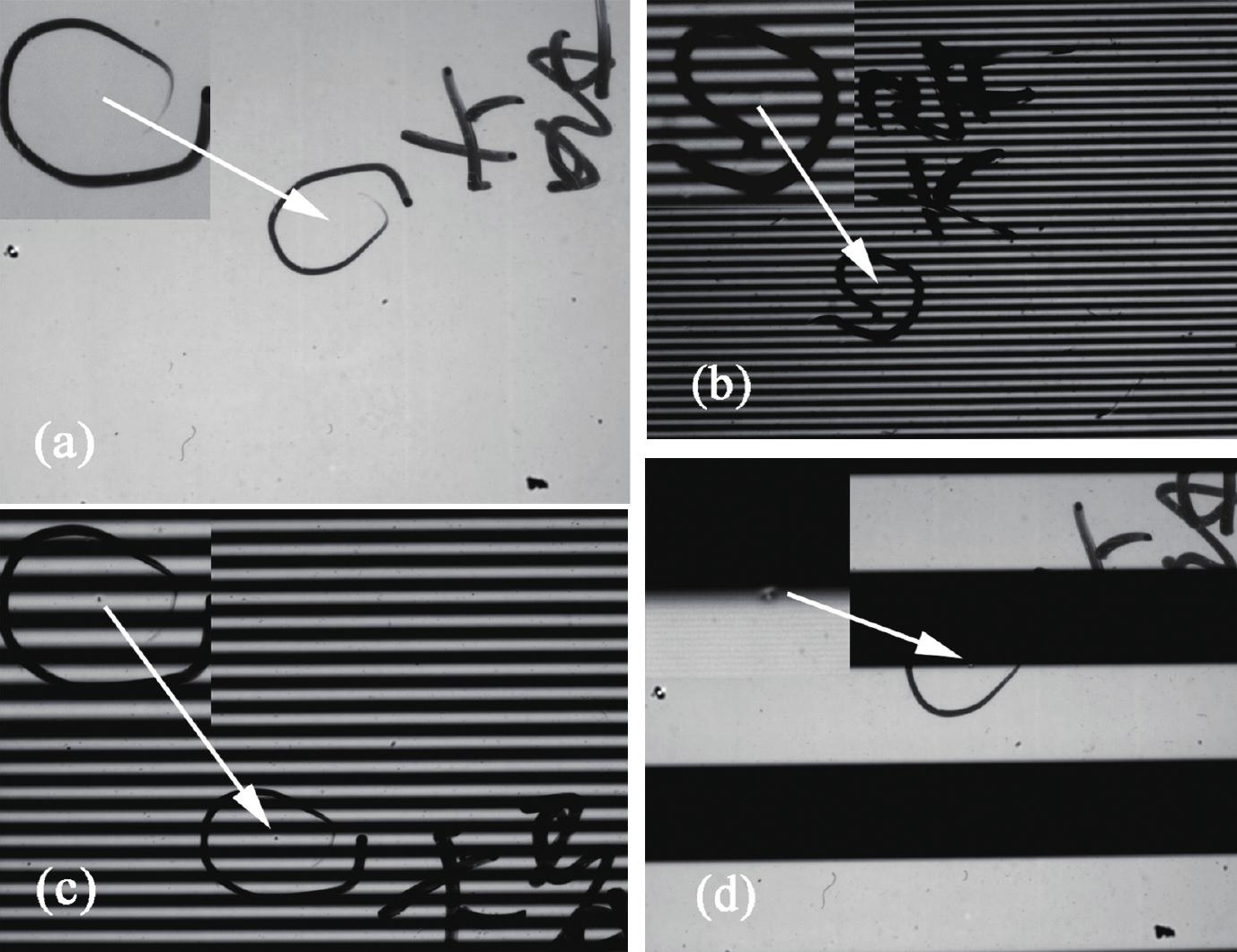




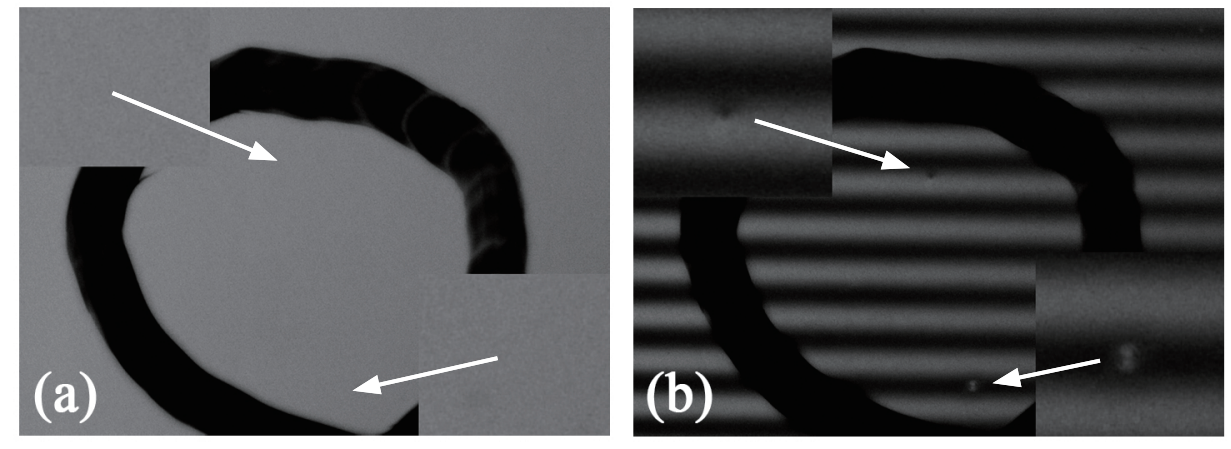

(c)
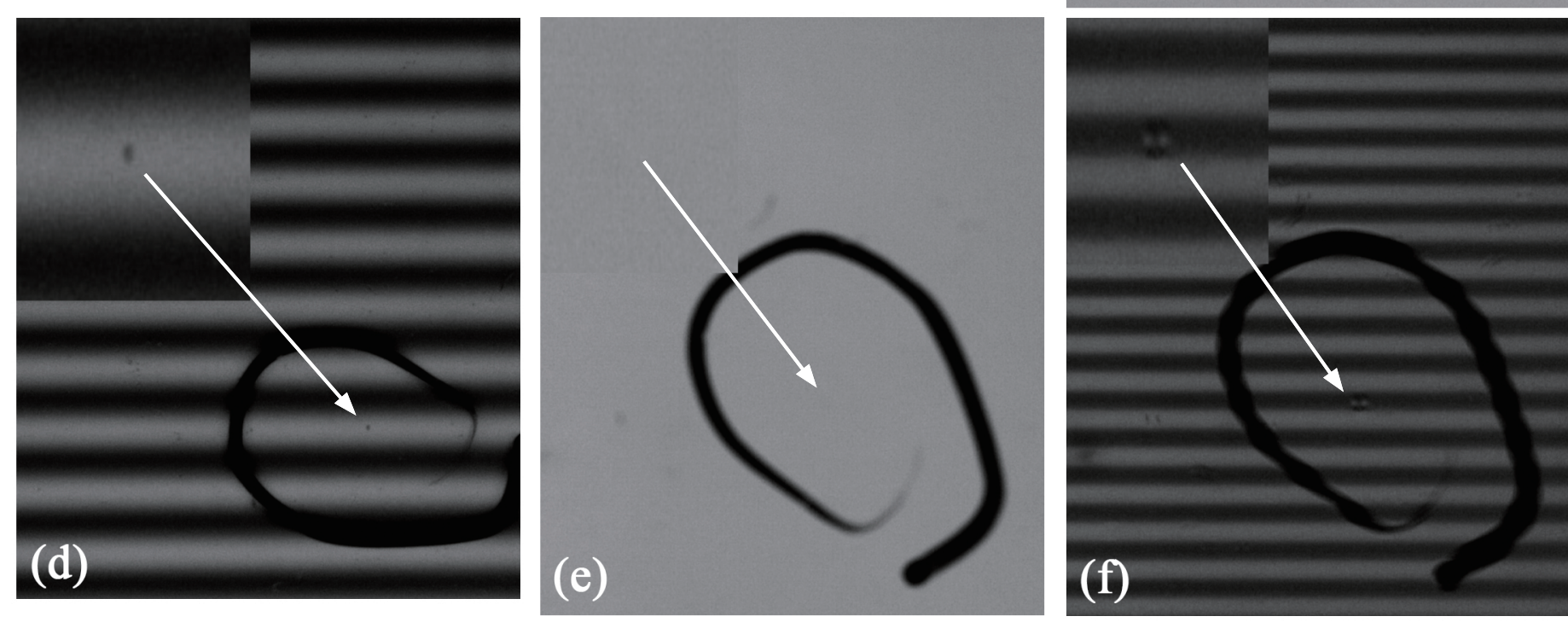

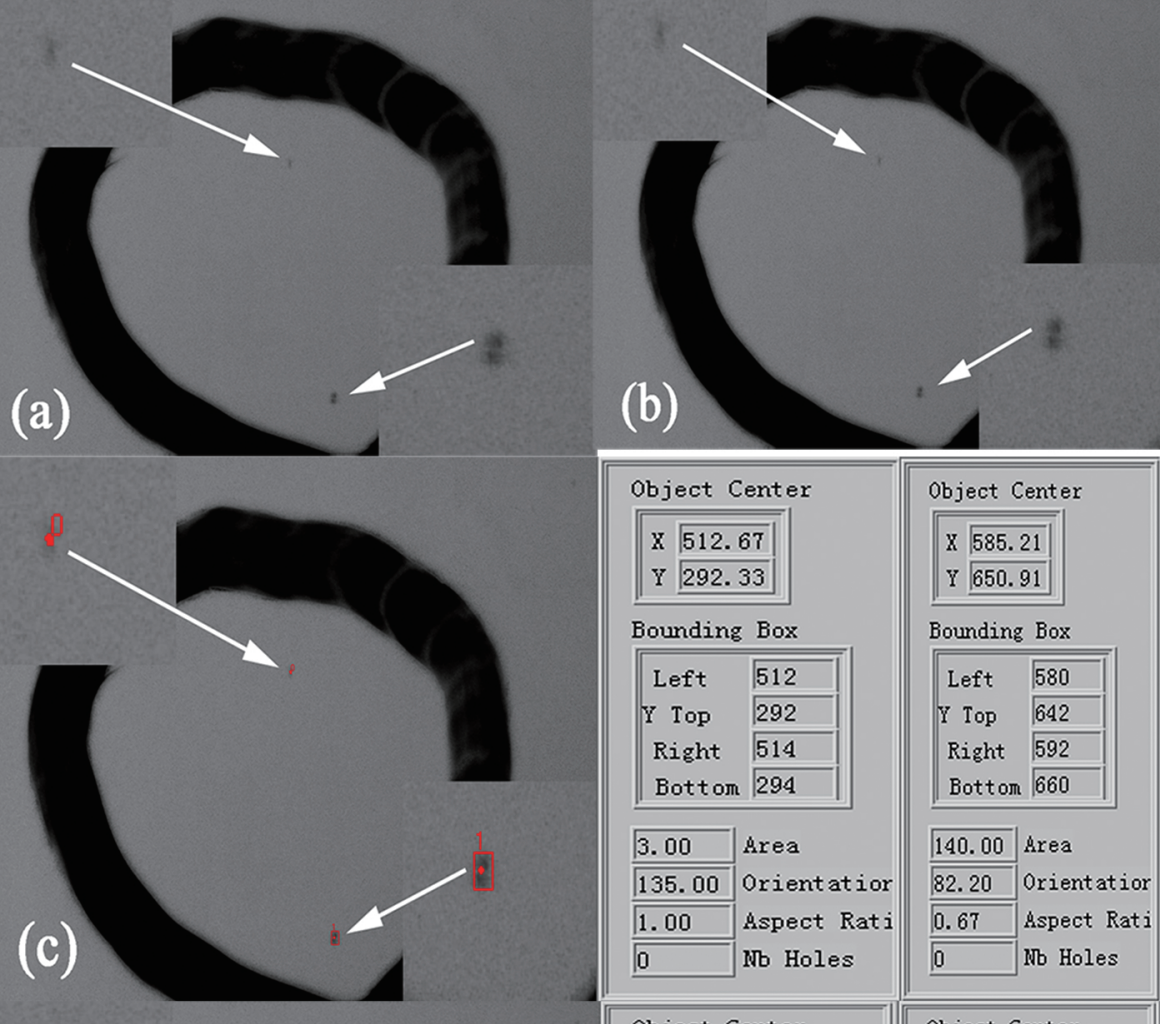

Object Center

\begin{tabular}{|l|l|}
\hline $\mathrm{X}$ & $\mathbf{5 1 2 . 6 7}$ \\
$\mathrm{Y}$ & 292.33 \\
\hline
\end{tabular}

Bounding Box

\begin{tabular}{|c|c|}
\hline Left & 512 \\
\hline Y Top & 292 \\
\hline Right & 514 \\
\hline Bott om & 294 \\
\hline
\end{tabular}

\begin{tabular}{|c|c|}
\hline 3.00 & Area \\
\hline 135.00 & Orientatior \\
\hline 1.00 & Aspect Rati \\
\hline 0 & No Holes \\
\hline
\end{tabular}

Object Center

\begin{tabular}{|l|l|}
\hline $\mathrm{X}$ & 512.67 \\
$\mathrm{Y}$ & 292.33 \\
\hline
\end{tabular}

Bounding Box

\begin{tabular}{||l|l|}
\hline Left & 512 \\
\hline $\begin{array}{l}\text { Y Top } \\
\text { Right }\end{array}$ & 292 \\
\hline Bottom & 294 \\
\hline
\end{tabular}

\begin{tabular}{|c|c|}
\hline 3.00 & Sxea \\
\hline 135.00 & Orientatior \\
\hline 1.00 & Aspect Rati \\
\hline 0 & Wb Holes \\
\hline
\end{tabular}

Object Center

\begin{tabular}{|l|l|}
\hline $\mathrm{X}$ & $\mathbf{5 8 5 . 2 1}$ \\
$\mathrm{Y}$ & $\mathbf{6 5 0 . 9 1}$ \\
\hline
\end{tabular}

Bounding Box

\begin{tabular}{|c|c|}
\hline Left & 580 \\
\hline I Top & 642 \\
\hline Right & 592 \\
\hline Bottom & 660 \\
\hline
\end{tabular}

\begin{tabular}{|c|c|}
\hline 140.00 & Area \\
\hline 82.20 & Orientatior \\
\hline 0.67 & Aspect Rati \\
\hline 0 & Wb Holes \\
\hline
\end{tabular}

object Center

$$
\begin{array}{|l|l|}
\hline \mathrm{X} & 585.29 \\
\mathrm{Y} & 650.93 \\
\hline
\end{array}
$$

\begin{tabular}{|c|c|}
\hline Left & 580 \\
\hline Y Top & 642 \\
\hline Right & 592 \\
\hline Bott om & 660 \\
\hline 137.00 & \multirow{4}{*}{$\begin{array}{l}\text { Area } \\
\text { Orientatior } \\
\text { Aspect Rati } \\
\text { Nb Holes }\end{array}$} \\
\hline 82.58 & \\
\hline 0.67 & \\
\hline 0 & \\
\hline
\end{tabular}

Bounding Box 


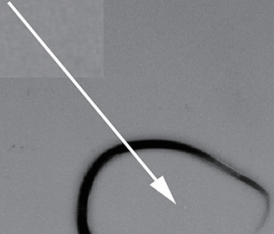

(a)

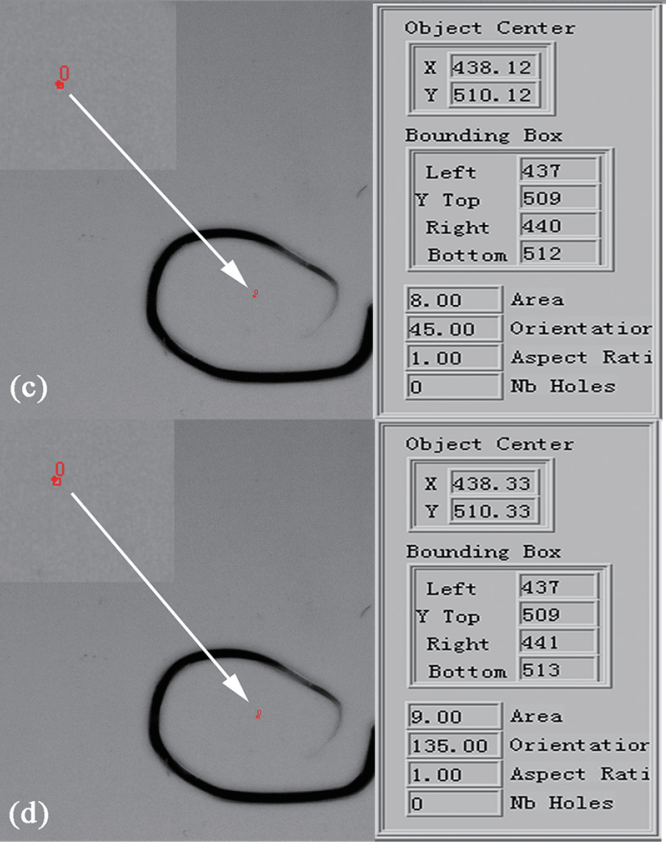

(b)

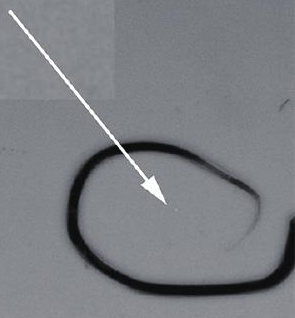$$
\text { | }
$$ 
